Sociodemographic Correlates of Sexlessness Among American Adults and Associations with Self-Reported Happiness Levels:

Evidence from the U.S. General Social

Survey

Jean H. Kim, Wilson S. Tam \& Peter Muennig

\title{
Archives of Sexual Behavior
}

The Official Publication of the

International Academy of Sex Research

ISSN 0004-0002

Arch Sex Behav

DOI 10.1007/s10508-017-0968-7

\section{Archives of}

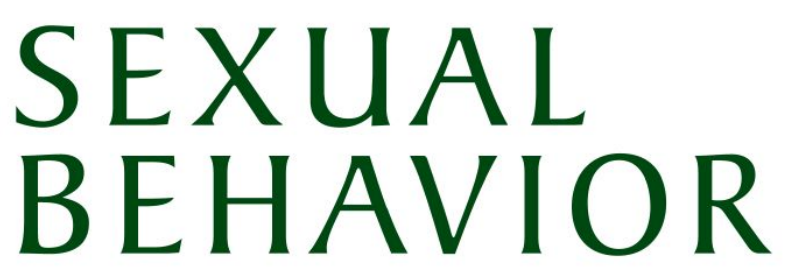

The Official Publication of the

International Academy of Sex Research

\section{管 Springer}

Available

online

wwwspringerlink.com 
Your article is protected by copyright and all rights are held exclusively by Springer Science +Business Media New York. This e-offprint is for personal use only and shall not be selfarchived in electronic repositories. If you wish to self-archive your article, please use the accepted manuscript version for posting on your own website. You may further deposit the accepted manuscript version in any repository, provided it is only made publicly available 12 months after official publication or later and provided acknowledgement is given to the original source of publication and a link is inserted to the published article on Springer's website. The link must be accompanied by the following text: "The final publication is available at link.springer.com". 


\title{
Sociodemographic Correlates of Sexlessness Among American Adults and Associations with Self-Reported Happiness Levels: Evidence from the U.S. General Social Survey
}

\author{
Jean H. Kim ${ }^{1}$ Wilson S. Tam ${ }^{2} \cdot$ Peter Muennig ${ }^{3}$
}

Received: 12 May 2015 / Revised: 17 February 2017 / Accepted: 20 February 2017

(C) Springer Science+Business Media New York 2017

\begin{abstract}
Although sexual activity is commonly believed to be a key component of emotional well-being, little is known about the factors associated with the absence of sexual activity or its associations with self-reported happiness. Using the U.S. General Social Survey-National Death Index 2008 dataset, a series of nationally representative surveys (1988-2002), this study analyzed the sociodemographic and lifestyle factors associated with past-year sexlessness and self-reported happiness among American adults $(n=17,744)$. After adjustment for marital status, there were no significant time trends evident in the proportion of American adults reporting past-year sexlessness. Among participants (age $=18-89$ years), $15.2 \%$ of males and $26.7 \%$ of females reported past-year sexlessness while $8.7 \%$ of males and $17.5 \%$ of females reported no sex for 5 years or more. For both genders, past-year sexlessness was most strongly associated with older age and being currently non-married in the multivariable models. Among males, the multivariable analysis also showed that sexlessness was associated with providing less than $20 \%$ of the household income (OR 2.27). In female participants, sexlessness was associated with very low income, poor health, lower financial satisfaction, absence of children, and having conservative sexual attitudes (OR 1.46-3.60). For both genders, Black race was associated with a much lower likelihood of sexlessness among currently non-married adults. The purported detrimental impact of sexlessness
\end{abstract}

Peter Muennig

pm124@columbia.edu

1 The School of Public Health and Primary Care, The Chinese University of Hong Kong, Prince of Wales Hospital, Shatin, New Territories, Hong Kong

2 Alice Lee Centre for Nursing Studies, National University of Singapore, Singapore, Singapore

3 The Mailman School of Public Health, Columbia University, New York, NY 10032, USA on self-reported happiness levels was not evident in this large, nationally representative study after adjusting for sociodemographic factors. Sexless Americans reported very similar happiness levels as their sexually active counterparts.

Keywords Sexual activity - Abstinence - Virginity . Asexuality $\cdot$ Celibacy $\cdot$ DSM-5

\section{Introduction}

Human sexuality research has spanned a wide spectrum of biomedical and sociomedical disciplines, but there have been surprisingly few large-scale studies exploring the factors associated with the absence of sexual activity and its effects on well-being. Although research in this area has been advanced in recent years by interest in the phenomenon of asexuality, or lack of sexual attraction to others (Bogaert, 2004, 2015; Hoglund, Jern, Sandnabba, \& Santtila, 2014), previous population-based studies of sexual activity have primarily been conducted to provide prevalence estimates of sexual behaviors (e.g., age of first coitus, presence of multiple sex partners, and engagement in homosexual sex) (Centers for Disease Control, 2002; Finer, 2007; Herbenick et al., 2010; Sandfort, Orr, Hirsch, \& Santelli, 2008; Smith, 1991, 2006; Turner, Danella, \& Rogers, 1995) for the purposes of tracking behavioral risk factors and informing policy. The primary shortcoming of these larger national surveys is that sexual activity is typically only examined in association with basic demographic characteristics and health risk behaviors, such as condom use and alcohol consumption. Moreover, due to the lack of a scientific consensus on the definition of sexlessness, such studies have varied widely in the duration of sexual inactivity from as little as 1-3 months (Herbenick et al., 2010; Moriki, Hayashi, \& Matsukura, 2015) to lifetime abstinence (Chou, Ng, \& Yu, 2014; Eisenberg, Shindel, Smith, Lue, \& Walsh, 2009; Haydon, Cheng, Herring, McRae, \& Halpern, 2014). These larger, 
population-based studies also tend not to examine links between sexuality and mental health, and often neglect contextual factors, such as household composition and the presence of life stressors.

In recent years, there has been increased research interest in positive psychology and subjective well-being (Seligman \& Csikszentmihalyi, 2000; Seligman, Steen, Park, \& Peterson, 2005). Much research has focused upon the common aspects of subjective well-being or self-reported happiness such as personality traits, social class, environmental aspects. Relatedness, or feelings of closeness with others, has been shown to be an integral aspect of positive well-being according to social determination theory (Ryan \& Deci, 2000). Conventional wisdom among health researchers has thereby been that safe, consensual sex is essential for positive mood and general psychological health (Heiman et al., 2011). Hence, low levels of sexual activity among physically healthy adults have often been viewed as a marker of poor emotional and sexual well-being (Blanchflower \& Oswald, 2004; Shreiner-Engel $\&$ Schiavi, 1986). Moreover, there has been a long-standing belief in popular psychology that sexlessness is symptomatic of longterm relationship problems (Schnarch \& Maddock, 2003; WeinerDavis, 2003), which are, in turn, correlated with poorer mental health outcomes (Prigerson, Maciejewski, \& Rosenheck, 1999).

Despite clear implications for well-being research, prior investigations of sexual activity levels and mental health, however, have mainly examined associations in the context of sexual dysfunctions (Laumann, Glasser, Neves, \& Moreira, 2009; Laumann et al., 2005; Marsiglio \& Donnelly, 1991; Nicolosi et al., 2005; Shifren et al., 2008), in the chronically ill (Marsiglio \& Donnelly, 1991; Pangman \& Seguire, 2000), younger people (Eisenberg, Shindel, Smith, Breyer, \& Lipshultz, 2010; Rasberry \& Goodson, 2009), in substance abusing populations (Nettleman, Ingersoll, \& Ceperich, 2006), and in older adults (DeLamater \& Moorman, 2007; Lindau \& Gavrilova, 2010; Lindau et al., 2007; Matthias, Lubben, Atchison, \& Schweitzer, 1997; Nicolosi et al., 2006; Palacios-Cena et al., 2012; Patel, Gillespie, \& Foxman, 2003; Schick et al., 2010; Wong, Leung, \& Woo, 2009).

Since sexual abstinence can be presumed to be largely involuntary in most of these studied populations, sexual inactivity has, not surprisingly, been correlated with poorer mental health (Dunn, Croft, \& Hackett, 1999; Rosen et al., 2009; Shifren, Monz, Russo, Segreti, \& Johannes, 2008). Studies that have investigated the importance of sexual well-being to general happiness have been limited to sexually active participants (Blanchflower \& Oswald, 2004). Although contextual factors, clinical factors, and lifestyle variables were often included in smaller sexual behavior surveys, these smaller studies were often conducted on non-representative samples that limited the generalizability of the results. The ideal study would be nationally representative and contain a wide array of psychosocial variables.

\section{Aims}

In order to address the dearth of scientific literature on the absence of sexual inactivity in the general population of healthy adults, the current study examined short-term and long-term sexual inactivity by estimating the prevalence of past-year and past 5-year sexlessness, defined as the absence of sexual partners, among a representative sample of adults in the U.S. This study also explored the sociodemographic, attitudinal, and lifestyle factors that were associated with past-year sexlessness. Finally, we investigated whether current happiness levels were associated with past-year sexlessness. Whether lack of sexual activity is, in fact, associated with lower subjective well-being in the general population bears importance for further research on human well-being and should be examined in order to inform clinical practice.

\section{Method}

\section{Participants}

The study population was drawn from the General Social Survey (GSS) linked to the National Death Index (the 2008 GSS-NDI) (Muennig, Johnson, Kim, Smith, \& Rosen, 2011). The GSS is a serial cross-sectional survey conducted on a nationally representative sample of non-institutionalized U.S. adults over the age of 18 years. The study excludes those residing in long-term care facilities, school dormitories, religious institutes, military installations, and correctional facilities. Similar to other national surveys, the GSS only covers the non-institutionalized, civilian population due to the difficulty of obtaining special authorizations for conducting surveys within institutions such as the military or prison system.

\section{Procedure}

The face-to-face surveys have been conducted by the National Opinion Research Center almost every year since 1972. Between 1978 and 2002, the GSS has interviewed 55,087 participants with an overall response rate of over $80 \%$, with extensive follow-up done to ensure non-responders are similar to responders. Questions related to sexual behaviors, however, have only been included since 1988 and this analysis thereby only examined participants interviewed in 1988-1991, 1993, 1994, 1996, 1998, 2000, and 2002 $(n=17,728)$.

\section{Measures}

The data analyzed in this survey include the following demographic items: gender, marital status (currently married, never been married, divorced/separated, widowed), age in years, educational 
attainment ( 11 years or less, 12 years, $13-16$ years, $17+$ years), race (White, Black, other), presence of children (yes, no), household income group based upon inflation-adjusted income (adjusted for year 2000 dollar equivalents). The study recoded household into six levels based upon the distribution of the household income $(<\$ 15 \mathrm{~K}$ to $75 \mathrm{~K}+$ /year) to allow sufficient sample sizes to conduct multivariate analysis. We recoded the participant's contribution to household income $(<20,20-39,40-59,60-79$ and $>80 \%)$ to investigate the relationship between household contributions and sexlessness. Additionally, this study instrument asked participants about their current self-rated physical health on a 4-point Likert scale (excellent, good, fair, and poor). The instruments asked participants about their current level of happiness (Taken all together, how would you say things are these days, would you say that you are very happy, pretty happy, or not too happy?).

Participants' job/work satisfaction and financial satisfaction were asked (satisfied, more or less satisfied, and dissatisfied). Sexual orientation was classified as heterosexual only or having some homosexual experience since age 18. We examined the levels of social engagement by classifying the number of times the participant socialized with friends and neighbors into three levels of socializing frequency (at least several times a month, once a month to several times per year, once a year or less). This study also examined conservative sexual beliefs measured as: attitudes toward homosexuality, teen sex, premarital sex, and extramarital sex (always wrong, almost always wrong, sometimes wrong, not wrong at all). The sexual attitudes variables were combined into an additive scale measuring sexual conservatism (scored 0-16) and trichotomized into three levels of increasing sexual conservatism. The scale had internal reliability (Cronbach's alpha $=.65$ ) above the minimally acceptable threshold (DeVellis, 1991).

The participant's sexual activity was examined by two openended items that asked how many sex partners the participant had in the preceding year and in the preceding five years. Participants who reported no sexual partners were recoded as past-year sexless (Yes/No) and past 5-year sexless (Yes/No), respectively. The item did not further inquire as to the type of sexual activity with the other person or whether the sexual activity was voluntary or not.

\section{Data Analysis}

Background characteristics of the study sample were summarized after stratification by sexual activity status (Table 1). Preliminary analyses noted no statistically significant period effects in the overall study sample after adjusting for marital status, so data across all surveys were aggregated for further analyses.

The study first examined the prevalence of sexlessness after stratification by gender, marital status, and race to examine sexless status in the US population by these broad sociodemographic classi= fications. The odds ratios for sexless status were calculated(White race as referent group) after adjusting for older age ( $65+$ years) and household income level (Table 2) which were identified as the major confounding factors in the preliminary unadjusted analyses.
The study then examined the prevalence of sexlessness by demographic, health, lifestyle, and attitudinal factors (Tables 3,4). The unadjusted odds ratios and $95 \%$ confidence intervals $(\mathrm{CI})$ were estimated to identify factors that were associated with past-year sexlessness. All sociodemographic variables that were marginally significant in the unadjusted analyses $(p<.20)$ were used as candidate variables for the sociodemographic multivariable stepwise logistic regression of sexless status. A second multivariable logistic regression model (full model) was estimated for past-year sexless status using sociodemographic, attitudinal, and lifestyle variables that were marginally significant in the unadjusted analyses $(p<$ .20) as candidate variables. All multivariable models included significant interaction terms of main effect variables.

After stratifying by marital subgroup, multinomial logistic regression analyses were conducted to examine the association with past-year sexlessness and the following outcome categories (very happy $=$ referent, pretty happy, and not too happy). In order to avoid confounding bias introduced by poor health status in the association between sexual activity and happiness (Lindau et al., 2007; Schick et al., 2010), we restricted the analysis to those with "excellent," "good," or "fair" health. The odds ratios and 95\% CI were reported after adjusting for the following potential confounding factors: age, household income levels, educational attainment, race, and level of social engagement. Since participants were asked about their current happiness levels, only associations with past-year sexlessness were examined. Statistical significance was set at $p<.05$. SPSS version 16.0 was used for descriptive analyses. SAS version 9.2 was used for multivariable analysis.

\section{Results}

The background characteristics of the study sample are shown in Table 1 . The study sample included adults $18-89$ years of age $(M=$ 45 years) at the time of the survey whose year 2000 inflation-adjusted annual household incomes ranged from $\$ 486$ to $\$ 166,419$ $($ median $=\$ 36,883)$. Of the study sample, $21.5 \%$ reported not having sexual activity in the year preceding the survey and $13.6 \%$ of the sample did not have sex for five years or more. As compared with their sexually active counterparts, past-year sexlessness was more prevalent in females, Whites, the elderly, currently unmarried individuals, those who were religiously observant, and those with lower SES. Sexless adults were also more likely to have poorer health and more conservative sexual attitudes $(p<.05)$. The majority of those who reported past-year sexlessness also reported past 5-year sexlessness, and the same significant trends were noted with background factors. There were no significant time trends in the proportion of sexless adults across the study period after adjusting for marital status.

The prevalence of past-year and past 5-year sexlessness stratified by sex, marital status, and race is shown in Table 2 . Never-married, divorced/separated, and widowed Black males showed lower likelihood of sexlessness as compared to their White counterparts, 
Table 1 Background characteristics of the General Social Survey Sample (1988-2002)

\begin{tabular}{|c|c|c|c|c|c|}
\hline & $\begin{array}{l}\text { Total sample } \\
(n=17,744)(\%)\end{array}$ & $\begin{array}{l}\text { Sex in past year } \\
(n=13,928)(\%)\end{array}$ & $\begin{array}{l}\text { Past-year sexless } \\
(n=3816)(\%)\end{array}$ & $\begin{array}{l}\text { Past 5-year sex } \\
(n=11,711)(\%)\end{array}$ & $\begin{array}{l}\text { Past 5-year sexless } \\
(n=1851)(\%)\end{array}$ \\
\hline \multicolumn{6}{|l|}{ Gender $^{\mathrm{a}, \mathrm{b}}$} \\
\hline Male & 43.6 & 47.0 & 30.9 & 46.3 & 28.0 \\
\hline Female & 56.4 & 53.0 & 69.1 & 53.7 & 72.0 \\
\hline \multicolumn{6}{|l|}{ Age at time of survey ${ }^{\mathrm{a}, \mathrm{b}}$} \\
\hline$<25$ & 9.9 & 10.8 & 6.4 & 9.8 & 7.6 \\
\hline 25-34 year & 22.6 & 26.6 & 7.8 & 25.4 & 5.6 \\
\hline 35-44 year & 23.4 & 26.9 & 10.5 & 26.4 & 7.3 \\
\hline $45-54$ year & 16.6 & 17.5 & 13.0 & 18.7 & 10.3 \\
\hline $55-64$ year & 11.0 & 10.0 & 14.6 & 10.4 & 13.6 \\
\hline $65-74$ year & 9.8 & 6.2 & 23.1 & 6.7 & 24.2 \\
\hline $75+$ year & 6.8 & 2.0 & 24.6 & 2.6 & 31.4 \\
\hline \multicolumn{6}{|l|}{$\operatorname{Race}^{\mathrm{a}, \mathrm{b}}$} \\
\hline White & 82.4 & 81.9 & 84.3 & 81.6 & 84.6 \\
\hline Black & 12.6 & 12.8 & 11.8 & 13.0 & 11.3 \\
\hline Other race & 5.1 & 5.4 & 3.9 & 5.5 & 4.1 \\
\hline \multicolumn{6}{|l|}{ Years of education $^{\mathrm{a}, \mathrm{b}}$} \\
\hline 11 years or less & 18.1 & 15.3 & 28.2 & 14.3 & 29.0 \\
\hline High school graduate & 30.5 & 30.4 & 30.8 & 30.0 & 30.9 \\
\hline 13-16 yrs (some college) & 40.4 & 42.6 & 32.3 & 43.6 & 30.9 \\
\hline 17+ years (post-college) & 11.1 & 11.7 & 8.8 & 12.1 & 9.2 \\
\hline \multicolumn{6}{|l|}{ Household income $e^{\mathrm{a}, \mathrm{b}, \mathrm{c}}$} \\
\hline$<\$ 20,000 /$ year & 26.2 & 21.0 & 46.5 & 21.7 & 49.5 \\
\hline 20,000-44,999/year & 33.1 & 33.1 & 33.2 & 34.0 & 31.9 \\
\hline $45,000-69,999 /$ year & 21.3 & 23.6 & 12.7 & 22.6 & 12.0 \\
\hline $70,000 /$ year & 19.3 & 22.4 & 7.6 & 21.7 & 6.6 \\
\hline \multicolumn{6}{|l|}{ Marital status ${ }^{\mathrm{a}, \mathrm{b}}$} \\
\hline Married & 50.2 & 59.6 & 15.6 & 54.9 & 13.5 \\
\hline Never married & 22.2 & 21.5 & 25.1 & 22.4 & 26.4 \\
\hline Divorced/separated & 18.2 & 16.6 & 24.0 & 18.6 & 20.2 \\
\hline Widowed & 9.4 & 2.3 & 35.4 & 4.0 & 39.8 \\
\hline \multicolumn{6}{|l|}{ Attend religious services? $?^{\mathrm{a}, \mathrm{b}}$} \\
\hline Never & 18.8 & 18.7 & 19.4 & 19.5 & 18.8 \\
\hline Less than weekly & 46.1 & 49.4 & 34.5 & 28.7 & 32.5 \\
\hline At least weekly & 35.0 & 32.0 & 46.0 & 31.8 & 48.8 \\
\hline \multicolumn{6}{|l|}{ Self-reported health status ${ }^{\mathrm{a}, \mathrm{b}}$} \\
\hline Excellent/good health & 78.5 & 82.2 & 65.1 & 81.2 & 64.2 \\
\hline Fair/poor health & 21.5 & 17.8 & 34.9 & 18.8 & 35.2 \\
\hline \multicolumn{6}{|l|}{ Agree with the following ${ }^{\mathrm{d}}$} \\
\hline Premarital sex is wrong ${ }^{a, b}$ & 35.1 & 30.0 & 54.0 & 30.4 & 61.6 \\
\hline Extramarital sex is wrong ${ }^{\mathrm{a}, \mathrm{b}}$ & 91.9 & 91.5 & 93.3 & 91.6 & 93.9 \\
\hline Teen sex is wrong ${ }^{a, b}$ & 86.7 & 85.2 & 92.1 & 85.7 & 92.7 \\
\hline Homosexual sex is wrong ${ }^{a, b}$ & 69.3 & 66.8 & 78.4 & 70.3 & 84.2 \\
\hline
\end{tabular}


Table 1 continued

\begin{tabular}{|c|c|c|c|c|c|}
\hline & $\begin{array}{l}\text { Total sample } \\
(n=17,744)(\%)\end{array}$ & $\begin{array}{l}\text { Sex in past year } \\
(n=13,928)(\%)\end{array}$ & $\begin{array}{l}\text { Past-year sexless } \\
(n=3816)(\%)\end{array}$ & $\begin{array}{l}\text { Past 5-year sex } \\
(n=11,711)(\%)\end{array}$ & $\begin{array}{l}\text { Past 5-year sexless } \\
(n=1851)(\%)\end{array}$ \\
\hline \multicolumn{6}{|c|}{ GSS Study Cohort $^{\mathrm{e}, \mathrm{f}}$} \\
\hline 1988-1994 & 48.3 & 48.1 & 48.9 & 34.1 & 34.5 \\
\hline 1996-2002 & 51.7 & 51.9 & 51.1 & 65.9 & 65.5 \\
\hline
\end{tabular}

${ }^{\mathrm{a}} p<.05$ for past-year sexless

b $p<.05$ for past 5 -year sexless

c Inflation adjusted to year 2000

d Responded that that the action was "Always" or "Almost Always" wrong

e Not significant at $p<.05$ for past-year sexless

${ }^{\mathrm{f}}$ Not significant at $p<.05$ for past 5 -year sexless

Table 2 Prevalence of past-year and past 5-year sexlessness by gender, marital status, and race of American adults (18-89 years of age)

\begin{tabular}{|c|c|c|c|c|c|c|c|c|}
\hline \multirow{2}{*}{$\begin{array}{l}\text { Current } \\
\text { marital } \\
\text { status }\end{array}$} & \multicolumn{4}{|l|}{ Males } & \multicolumn{4}{|l|}{ Females } \\
\hline & $\begin{array}{l}\text { Males } \\
(n)\end{array}$ & $\begin{array}{l}\text { Past-year } \\
\text { sexless/past } \\
\text { 5-year sexless }(\%)\end{array}$ & $\begin{array}{l}\text { OR }(95 \% \mathrm{CI})^{\mathrm{a}} \\
\text { past-year } \\
\text { sexless }\end{array}$ & $\begin{array}{l}\text { OR }(95 \% \mathrm{CI})^{\mathrm{a}} \\
\text { past 5-year } \\
\text { sexless }\end{array}$ & $\begin{array}{l}\text { Females } \\
(n)\end{array}$ & $\begin{array}{l}\text { Past-year } \\
\text { sexless/past } \\
\text { 5-year sexless }(\%)\end{array}$ & $\begin{array}{l}\text { OR }(95 \% \mathrm{CI})^{\mathrm{a}} \\
\text { past-year } \\
\text { sexless }\end{array}$ & $\begin{array}{l}\text { OR }(95 \% \mathrm{CI})^{\mathrm{a}} \\
\text { past 5-year } \\
\text { sexless }\end{array}$ \\
\hline \multicolumn{9}{|l|}{ Married } \\
\hline White & 3673 & $6.8 / 3.7$ & 1.00 & 1.00 & 4120 & $6.5 / 3 / 6$ & 1.00 & 1.00 \\
\hline Other race & 194 & $6.2 / 4.4$ & ns & ns & 227 & $3.5 / 2.9$ & ns & ns \\
\hline Black & 334 & $9.0 / 4.8$ & $\begin{array}{l}1.66 \\
\quad(1.02-2.70)^{*}\end{array}$ & $\mathrm{~ns}$ & 351 & $8.5 / 4.8$ & $\mathrm{~ns}$ & ns \\
\hline \multicolumn{9}{|c|}{ Never married } \\
\hline White & 1625 & $25.4 / 16.2$ & 1.00 & 1.00 & 1265 & $28.9 / 19.9$ & 1.00 & 1.00 \\
\hline Other race & 143 & $24.5 / 15.7$ & $\mathrm{~ns}$ & ns & 147 & $23.8 / 17.5$ & $\mathrm{~ns}$ & ns \\
\hline Black & 247 & $8.5 / 5.7$ & $\begin{array}{l}0.31 \\
(0.20-0.51)^{* *}\end{array}$ & $\begin{array}{l}0.35 \\
(0.18-0.67)^{*}\end{array}$ & 518 & $16.8 / 8.6$ & $\begin{array}{l}0.58 \\
(0.43-0.79)^{*}\end{array}$ & $\begin{array}{l}0.39 \\
(0.24-0.62)^{* * *}\end{array}$ \\
\hline \multicolumn{9}{|c|}{ Divorced/separated } \\
\hline White & 989 & $20.6 / 8.0$ & 1.00 & 1.00 & 1556 & $35.8 / 20.3$ & 1.00 & 1.00 \\
\hline Other race & 47 & $17.0 / 2.6$ & ns & ns & 96 & $26.0 / 15.7$ & ns & $\mathrm{ns}$ \\
\hline Black & 172 & $12.8 / 5.6$ & $\begin{array}{l}0.55 \\
(0.33-0.92)^{*}\end{array}$ & ns & 364 & $26.9 / 14.8$ & $\begin{array}{l}0.67 \\
(0.50-0.89)^{* *}\end{array}$ & $\begin{array}{l}0.60 \\
(0.38-0.95)^{*}\end{array}$ \\
\hline \multicolumn{9}{|l|}{ Widowed } \\
\hline White & 258 & $64.0 / 40.8$ & 1.00 & 1.00 & 1131 & $88.2 / 69.1$ & 1.00 & 1.00 \\
\hline Other race & 9 & $33.3 / 20.0$ & $\mathrm{~ns}$ & ns & 33 & $72.7 / 47.6$ & ns & ns \\
\hline Black & 38 & $42.1 / 20.7$ & $\begin{array}{l}0.41 \\
(0.18-0.98)^{*}\end{array}$ & $\mathrm{~ns}$ & 204 & $71.1 / 55.8$ & $\begin{array}{l}0.34 \\
(0.21-0.53)^{* *}\end{array}$ & $\begin{array}{l}0.52 \\
(0.32-0.84)^{*}\end{array}$ \\
\hline
\end{tabular}

$* p<.05 ; * *<.001$

${ }^{a}$ Adjusted for income (0-19,999, 20 K-44,999, 50 K-69,999, 70 K) and age (elderly vs. not)

whereas married Black males had higher likelihood of sexlessness. Among females, Black race was significantly associated with lower likelihood of past-year and past 5-year sexlessness among nevermarried, divorced/separated, and widowed participants.

The proportion of participants who reported past-year and past 5-year sexlessness is shown by sociodemographic, attitudinal, and lifestyle factors among males (Table 3) and among females (Table 4). Among male participants, all sociodemographic factors except homosexual orientation were statistically significant with respect to associations with sexlessness in the unadjusted analyses. However, the full multivariable model of sociodemographic, lifestyle, and attitudinal factors showed that older age and not being 
Table 3 Factors associated with sexlessness among all males participants from the General Social Survey

\begin{tabular}{|c|c|c|c|c|c|}
\hline & $\begin{array}{l}\% \text { past- } \\
\text { year } \\
\text { sexless }\end{array}$ & $\begin{array}{l}\% 5 \text {-year } \\
\text { sexless }\end{array}$ & $\begin{array}{l}\text { Unadjusted model } \\
\text { for past-year sexlessness } \\
\text { OR }(95 \% \mathrm{CI})\end{array}$ & $\begin{array}{l}\text { Demographic and health } \\
\text { factors model for past-year } \\
\text { sexlessness OR }(95 \% \text { CI })\end{array}$ & $\begin{array}{l}\text { All factors model } \\
\text { for past-year sexless } \\
\text { OR }(95 \% \mathrm{CI})\end{array}$ \\
\hline Total male study sample & 15.2 & 8.7 & & & \\
\hline \multicolumn{6}{|l|}{ Age at time of survey } \\
\hline$<25$ & 16.2 & 13.6 & 1.00 & 1.00 & 1.00 \\
\hline $25-34$ y & 8.2 & 3.4 & $0.46(0.36-0.59)^{*}$ & $0.97(0.66-1.43)$ & $0.98(0.52-1.87)$ \\
\hline $35-44 y$ & 7.7 & 3.3 & $0.43(0.34-0.56)^{*}$ & $1.70(1.12-2.56) *$ & $1.78(0.90-3.53) \S$ \\
\hline $45-54$ y & 12.6 & 6.7 & $0.75(0.58-0.96)^{*}$ & $3.86(2.48-6.00)^{*}$ & $5.01(2.39-10.5)^{\ddagger}$ \\
\hline $55-64$ y & 15.0 & 6.8 & $0.91(0.70-1.19)$ & $3.23(1.95-5.35)^{*}$ & $2.64(1.00-6.97)^{*}$ \\
\hline $65+$ & 41.6 & 28.3 & $3.69(2.95-4.60)^{*}$ & $18.2(10.6-31.3)^{*}$ & $28.7(11.4-72.3)^{\ddagger}$ \\
\hline \multicolumn{6}{|l|}{ Years of education } \\
\hline $17+$ years (post-college) & 12.2 & 6.5 & 1.00 & Non-significant ${ }^{\mathrm{a}}$ & Non-significant ${ }^{\mathrm{b}}$ \\
\hline 13-16 years (college) & 12.9 & 7.1 & $1.06(0.86-1.32)$ & & \\
\hline HS diploma (12 years) & 15.1 & 8.6 & $1.28(1.02-1.60)^{*}$ & & \\
\hline 11 years or less & 23.2 & 15.3 & $2.17(1.73-2.73)^{*}$ & & \\
\hline \multicolumn{6}{|l|}{ Race } \\
\hline White & 15.8 & 9.1 & 1.00 & 1.00 & 1.00 \\
\hline Black & 11.2 & 6.0 & $0.68(0.54-0.85) \dagger$ & $1.58(0.73-3.43)$ & $3.81(1.29-11.3)^{*}$ \\
\hline Other race & 14.7 & 8.5 & $0.92(0.69-1.23)$ & $2.29(0.95-5.50)$ & $4.29(1.13-16.3)^{*}$ \\
\hline \multicolumn{6}{|l|}{ Marital status } \\
\hline Married & 6.9 & 3.8 & 1.00 & 1.00 & 1.00 \\
\hline Never married & 23.3 & 14.9 & $4.08(3.48-4.77)^{*}$ & $12.3(7.96-18.9)^{*}$ & $35.5(17.7-71.3)^{\ddagger}$ \\
\hline Divorced/separated & 19.4 & 7.4 & $3.23(2.68-3.89)^{*}$ & $6.06(4.16-8.82)^{*}$ & $11.8(5.93-23.6)^{\ddagger}$ \\
\hline Widowed & 60.3 & 37.9 & $20.4(15.8-26.5)^{*}$ & $18.5(10.3-33.2)^{*}$ & $68.9(23.9-198.6)^{*}$ \\
\hline \multicolumn{6}{|l|}{ Past-year household income ${ }^{* *}$} \\
\hline$\$ 75,000+/$ year & 7.2 & 4.1 & 1.00 & 1.00 & Non-significant $^{\mathrm{c}}$ \\
\hline$\$ 60 \mathrm{~K}-74,999 /$ year & 9.2 & 5.3 & $1.30(0.94-1.79)$ & $1.05(0.66-1.68)$ & \\
\hline$\$ 45 \mathrm{~K}-59,999 /$ year & 11.4 & 5.9 & $1.65(1.26-2.17)^{*}$ & $1.53(1.03-2.25) *$ & \\
\hline$\$ 30 \mathrm{~K}-44,999 /$ year & 13.2 & 7.3 & $1.95(1.52-2.51)^{*}$ & $1.47(1.01-2.11)^{*}$ & \\
\hline$\$ 15 \mathrm{~K}-29,999 /$ year & 20.4 & 11.0 & $3.29(2.60-4.16)^{*}$ & $1.60(1.10-2.32)^{*}$ & \\
\hline$<\$ 15 \mathrm{~K} /$ year & 29.1 & 18.8 & $5.28(4.14-6.74)^{*}$ & $1.59(1.03-2.45) *$ & \\
\hline \multicolumn{6}{|l|}{ Household income contribution } \\
\hline $80-100 \%$ & 11.3 & 5.1 & 1.00 & 1.00 & 1.00 \\
\hline $60-79 \%$ & 6.9 & 3.4 & $0.58(0.43-0.78)^{*}$ & $1.34(0.93-1.95)$ & $1.40(0.75-2.58)$ \\
\hline $40-59 \%$ & 7.2 & 3.3 & $0.61(0.47-0.79)^{*}$ & $1.10(0.77-1.57)$ & $1.03(0.56-1.92)$ \\
\hline $20-39 \%$ & 9.3 & 5.8 & $0.81(0.58-1.14)$ & $0.85(0.54-1.33)$ & $0.60(0.26-1.39)$ \\
\hline $0-19 \%$ & 21.6 & 18.2 & $2.16(1.60-2.93)^{*}$ & $2.10(1.40-3.18)^{*}$ & $2.27(1.19-4.31)^{*}$ \\
\hline \multicolumn{6}{|l|}{ Self-reported health } \\
\hline Excellent to fair health & 14.6 & 8.0 & 1.00 & 1.00 & Non-significant $^{\mathrm{d}}$ \\
\hline Poor health & 38.4 & 26.6 & $3.65(2.75-4.85)^{+}$ & $1.90(0.98-3.69) \S$ & \\
\hline \multicolumn{6}{|l|}{ Participant has children } \\
\hline No children & 20.7 & 12.8 & 1.00 & 1.00 & Non-significant ${ }^{e}$ \\
\hline Has children & 12.3 & 6.5 & $0.54(0.48-0.61)^{*}$ & $0.71(0.52-0.98) *$ & \\
\hline \multicolumn{6}{|l|}{ Marital status $\times$ race } \\
\hline Never married-Black & 8.4 & 5.7 & & $0.20(0.70-0.57) \dagger$ & $0.02(0.002-0.23) \dagger$ \\
\hline Never married-Other race & 28.1 & 15.7 & & $0.53(0.19-1.49)$ & $0.24(0.04-1.30) \S$ \\
\hline Divorced/Sep.—Black & 10.7 & 5.6 & & $0.59(0.21-1.63)$ & $0.19(0.04-0.88)^{*}$ \\
\hline Divorced/Sep.-Other race & 15.8 & 2.6 & & $0.59(0.16-2.16)$ & $0.30(0.03-2.68)$ \\
\hline Widowed-Black & 30.8 & 20.7 & & $0.06(0.01-0.65)^{*}$ & $\mathrm{NE}$ \\
\hline
\end{tabular}


Table 3 continued

\begin{tabular}{|c|c|c|c|c|c|}
\hline & $\begin{array}{l}\% \text { past- } \\
\text { year } \\
\text { sexless }\end{array}$ & $\begin{array}{l}\% \text { 5-year } \\
\text { sexless }\end{array}$ & $\begin{array}{l}\text { Unadjusted model } \\
\text { for past-year sexlessness } \\
\text { OR }(95 \% \mathrm{CI})\end{array}$ & $\begin{array}{l}\text { Demographic and health } \\
\text { factors model for past-year } \\
\text { sexlessness OR }(95 \% \mathrm{CI})\end{array}$ & $\begin{array}{l}\text { All factors model } \\
\text { for past-year sexless } \\
\text { OR }(95 \% \mathrm{CI})\end{array}$ \\
\hline Widowed-Other race & 0.0 & 20.0 & & $\mathrm{NE}$ & $\mathrm{NE}$ \\
\hline \multicolumn{6}{|l|}{ Psychosocial/lifestyle factors } \\
\hline \multicolumn{6}{|l|}{ Homosexual experience } \\
\hline Never since age 18 & 7.1 & NA & 1.00 & & Non-significant ${ }^{\mathrm{f}}$ \\
\hline Ever since age 18 & 8.7 & NA & $1.25(0.79-1.98)$ & & \\
\hline \multicolumn{6}{|l|}{ Current job/work satisfaction } \\
\hline Satisfied & 10.2 & 5.2 & 1.00 & & Non-significant ${ }^{\mathrm{g}}$ \\
\hline Neutral/not sure & 13.0 & 2.2 & $1.32(0.65-2.67)$ & & \\
\hline Dissatisfied & 11.7 & 7.2 & $1.17(0.94-1.46)$ & & \\
\hline \multicolumn{6}{|l|}{ Current financial satisfaction } \\
\hline Satisfied & 17.1 & 10.6 & 1.00 & & Non-significant $^{\mathrm{h}}$ \\
\hline More or less satisfied & 13.8 & 7.7 & $0.77(0.67-0.89) \dagger$ & & \\
\hline Not at all satisfied & 14.7 & 7.9 & $0.83(0.70-0.99) *$ & & \\
\hline \multicolumn{6}{|l|}{ Social engagement levels } \\
\hline At least several times/month & 14.2 & 8.4 & 1.00 & & Non-significant ${ }^{\mathrm{i}}$ \\
\hline Once/month-several times/yr & 13.1 & 6.6 & $0.91(0.76-1.09)$ & & \\
\hline Socialize once/year or less & 26.1 & 18.3 & $2.12(1.65-2.74)^{*}$ & & \\
\hline \multicolumn{6}{|l|}{ Sexual attitudes } \\
\hline Liberal attitudes & 11.7 & 5.8 & 1.00 & & 1.00 \\
\hline Moderate attitudes & 11.0 & 4.8 & $0.93(0.54-1.61)$ & & $1.07(0.52-2.18)$ \\
\hline Conservative attitudes & 17.2 & 11.5 & $1.56(0.92-2.65)^{\S}$ & & $(0.93-3.85)^{\S}$ \\
\hline
\end{tabular}

All statistically significant associations are shown in bold

$* p<.05 ;{ }^{\dagger} p<.01 ;{ }^{\dagger} p<.001 ;{ }^{\S} p<.10$

** Adjusted for Year 2000 equivalent value. NE non-estimatable due to small sample size

The following variables were dropped from the stepwise regression model. The odds ratios and $95 \%$ CI prior to exclusion in the multivariable models are shown

${ }^{\text {a }}$ Education: $13-16$ years $=0.98(0.69-1.39)$; high school diploma $=1.08(0.74-1.58) ; 11$ years or less $=1.01(0.65-1.57)$

b Education: $13-16$ years $=0.58(0.21-1.62)$; high school diploma $=0.65(0.21-2.02)$; 11 years or less $=0.35(0.06-1.94)$

${ }^{\mathrm{c}}$ Household income: $\$ 60 \mathrm{~K}-74,999=1.45(0.31-6.72) ; \$ 45 \mathrm{~K}-59,999=1.13(0.24-0.54) ; \$ 30 \mathrm{~K}-44,999=1.90(0.27-9.06) ; \$ 15 \mathrm{~K}-29,999=1.47$ $(0.21-10.1) ;<15 \mathrm{~K}=2.58(0.48-13.8)$

d Self-reported health: Poor health $=0.15(0.001-18.5)$

e Presence of children: Having children $=1.01(0.34-2.96)$

${ }^{\mathrm{f}}$ Homosexual experience: Gay/bisexual orientation $=2.51(0.73-8.66)$

g Job satisfaction: Neutral $=4.16(0.81-21.3)$; Dissatisfied $=0.89(0.51-1.55)$

${ }^{\mathrm{h}}$ Financial satisfaction: More or less satisfied $=1.29(0.49-3.36)$; Not at all satisfied $=1.54(0.51-4.68)$

${ }^{\mathrm{i}}$ Social engagement levels: Socialized $1 \times$ month-several times/year: $1.46(0.64-3.32)$; Socialized $1 \times /$ year or less $=1.16(0.18-7.35)$

married remained the factors most strongly associated with pastyear sexlessness. Lower contribution to household income $(<20 \%)$ showed moderate associations with past-year sexlessness, and conservative sexual attitudes showed marginally significant associations with past-year sexlessness. Among married males, non-Whites were more likely to be sexless but among non-married males, Blacks were much less likely to be sexless. Among females, the multivariable model limited to demographic and health factors showed associations that were similar to males. However, poor health and having no children were significantly associated with sexlessness while lower contribution to household income was not. When psychoso- cial factors were included as candidate variables, having a conservative sexual attitude and having low financial satisfaction were also significantly associated with past-year sexlessness $(p<.05)$. Never-married, divorced/separated, and widowed Blacks were much less likely to be sexless than their White female counterparts.

Finally, we explored whether self-reported happiness levels were associated with sexual activity for males and females without poor health (Table 5). After stratification by marital status, the multinomial odds ratios with being "Very Happy" as the referent outcome showed that past-year sexlessness was not associated with self-reported past-year happiness levels after adjusting for the 
Table 4 Factors associated with sexlessness among all females participants from the General Social Survey

\begin{tabular}{|c|c|c|c|c|c|}
\hline & $\begin{array}{l}\% \text { past- } \\
\text { year } \\
\text { sexless }\end{array}$ & $\begin{array}{l}\% 5 \text {-year } \\
\text { sexless }\end{array}$ & $\begin{array}{l}\text { Unadjusted model for } \\
\text { past-year sexlessness } \\
\text { odds ratio }(95 \% \mathrm{CI})\end{array}$ & $\begin{array}{l}\text { Demographic and health } \\
\text { factors model for past-year } \\
\text { sexlessness OR ( } 95 \% \text { CI) }\end{array}$ & $\begin{array}{l}\text { All factors model } \\
\text { for past-year sexless } \\
\text { OR }(95 \% \mathrm{CI})\end{array}$ \\
\hline Total female study sample & 26.3 & 17.4 & & & \\
\hline \multicolumn{6}{|l|}{ Age at time of survey } \\
\hline$<25$ & 11.8 & 8.6 & 1.00 & 1.00 & 1.00 \\
\hline 25-34 year & 6.8 & 3.3 & $0.54(0.42-0.71)^{*}$ & $0.97(0.69-1.36)$ & $0.78(0.47-1.32)$ \\
\hline 35-44 year & 11.2 & 4.9 & $0.95(0.75-1.20)$ & $2.99(2.11-4.23)^{*}$ & $1.74(0.99-3.07) \S$ \\
\hline 45-54 year & 20.4 & 9.2 & $1.92(1.52-2.42)^{*}$ & $7.25(5.04-10.4)^{*}$ & $5.34(2.99-9.54)^{\ddagger}$ \\
\hline 55-64 year & 38.9 & 25.2 & $4.77(3.78-6.01)^{*}$ & $22.4(15.1-33.1)^{*}$ & $15.9(8.48-29.6)^{\ddagger}$ \\
\hline $65+$ & 73.7 & 61.3 & $21.0(16.8-26.3)^{*}$ & $76.4(51.1-114.2)^{*}$ & $44.7(23.1-86.4)^{+}$ \\
\hline \multicolumn{6}{|l|}{ Years of education } \\
\hline $17+$ years (post-college) & 21.9 & 14.8 & 1.00 & Non-significant ${ }^{a}$ & Non-significant ${ }^{c}$ \\
\hline 13-16 years (college) & 20.6 & 12.4 & $0.93(0.78-1.10)$ & & \\
\hline HS diploma (12 years) & 26.2 & 17.8 & $1.27(1.07-1.50) \dagger$ & & \\
\hline 11 years or less & 41.0 & 31.1 & $2.48(2.07-2.96)^{*}$ & & \\
\hline \multicolumn{6}{|l|}{ Race } \\
\hline White & 27.1 & 18.2 & 1.00 & 1.00 & 1.00 \\
\hline Black & 25.1 & 15.4 & $0.90(0.79-1.02)$ & $1.50(0.86-2.62)$ & $2.13(0.89-5.08)$ \\
\hline Other race & 18.3 & 12.4 & $0.60(0.48-0.76)^{*}$ & $1.32(0.55-3.22)$ & $1.57(0.43-5.66)$ \\
\hline \multicolumn{6}{|l|}{ Marital status } \\
\hline Married & 6.5 & 3.7 & 1.00 & 1.00 & 1.00 \\
\hline Never married & 25.2 & 16.5 & $4.88(4.18-5.70)^{*}$ & $22.8(16.1-32.3)^{*}$ & $39.4(21.5-72.3)^{+}$ \\
\hline Divorced/separated & 33.7 & 19.1 & $7.36(6.34-8.53)^{*}$ & $15.7(12.0-20.51)^{*}$ & $23.1(14.6-36.5)^{*}$ \\
\hline Widowed & 85.2 & 66.6 & $83.4(69.0-100.8)^{*}$ & $31.7(23.3-43.1)^{*}$ & $49.5(28.9-85.0)^{+}$ \\
\hline \multicolumn{6}{|l|}{ Past-year household income ** } \\
\hline$\$ 75,000+/$ year & 8.5 & 4.3 & 1.00 & 1.00 & Non-significant $^{\mathrm{d}}$ \\
\hline$\$ 60 \mathrm{~K}-74,999 /$ year & 11.5 & 6.7 & $1.40(1.05-1.88)^{*}$ & $1.09(0.72-1.67)$ & \\
\hline$\$ 45 \mathrm{~K}-59,999 /$ year & 14.8 & 9.2 & $1.88(1.46-2.41)^{*}$ & $1.25(1.10-1.80)$ & \\
\hline$\$ 30 \mathrm{~K}-44,999 /$ year & 21.6 & 13.2 & $2.97(2.37-3.72)^{*}$ & $1.48(1.06-2.06)^{*}$ & \\
\hline$\$ 15 \mathrm{~K}-29,999 /$ year & 30.4 & 19.6 & $4.71(3.81-5.81)^{*}$ & $1.52(1.11-2.08)^{*}$ & \\
\hline$<\$ 15 \mathrm{~K} /$ year & 44.4 & 31.0 & $8.61(6.98-10.6)^{*}$ & $1.77(1.27-2.45) \dagger$ & \\
\hline \multicolumn{6}{|c|}{ Household income contribution } \\
\hline $80-100 \%$ & 25.9 & 14.0 & 1.00 & Non-significant $^{\mathrm{b}}$ & Non-significant $^{\mathrm{e}}$ \\
\hline $60-79 \%$ & 15.6 & 8.7 & $0.53(0.41-0.69)^{*}$ & & \\
\hline $40-59 \%$ & 8.6 & 4.6 & $0.27(0.22-0.34)^{*}$ & & \\
\hline $20-39 \%$ & 9.1 & 3.7 & $0.29(0.23-0.36)^{*}$ & & \\
\hline $0-19 \%$ & 10.7 & 7.4 & $0.34(0.27-0.43)^{*}$ & & \\
\hline \multicolumn{6}{|l|}{ Self-reported health } \\
\hline Excellent to fair health & 24.7 & 16.1 & 1.00 & 1.00 & 1.00 \\
\hline Poor health & 57.8 & 42.1 & $4.19(3.40-5.15)^{*}$ & $1.99(1.44-2.76)^{\ddagger}$ & $2.93(1.65-5.20)^{*}$ \\
\hline \multicolumn{6}{|l|}{ Participant has children } \\
\hline No children & 27.5 & 19.5 & 1.00 & 1.00 & 1.00 \\
\hline Has children & 25.9 & 16.8 & $0.21(0.83-1.02)$ & $0.56(0.45-0.81)^{*}$ & $0.46(0.31-0.69)^{+}$ \\
\hline \multicolumn{6}{|l|}{ Marital status $\times$ race } \\
\hline Never married-Black & 14.3 & 8.6 & & $0.37(0.19-0.72) \dagger$ & $0.16(0.05-0.46)^{\ddagger}$ \\
\hline Never married-Other race & 21.9 & 17.5 & & $0.98(0.35-2.72)$ & $0.79(0.17-3.74)$ \\
\hline Divorced/Sep.—Black & 24.2 & 14.8 & & $0.42(0.22-0.80) \dagger$ & $0.30(0.11-0.87)^{*}$ \\
\hline Divorced/Sep—Other race & 15.9 & 15.7 & & $0.65(0.21-1.97)$ & $0.15(0.02-1.11)$ \\
\hline Widowed-Black & 65.6 & 55.8 & & $0.29(0.13-0.61) \dagger$ & $0.10(0.03-0.37)^{+}$ \\
\hline
\end{tabular}


Table 4 continued

\begin{tabular}{|c|c|c|c|c|c|}
\hline & $\begin{array}{l}\% \text { past- } \\
\text { year } \\
\text { sexless }\end{array}$ & $\begin{array}{l}\% \text { 5-year } \\
\text { sexless }\end{array}$ & $\begin{array}{l}\text { Unadjusted model for } \\
\text { past-year sexlessness } \\
\text { odds ratio }(95 \% \mathrm{CI})\end{array}$ & $\begin{array}{l}\text { Demographic and health } \\
\text { factors model for past-year } \\
\text { sexlessness OR }(95 \% \mathrm{CI})\end{array}$ & $\begin{array}{l}\text { All factors model } \\
\text { for past-year sexless } \\
\text { OR }(95 \% \mathrm{CI})\end{array}$ \\
\hline Widowed-Other race & 68.8 & 47.6 & & $1.22(0.29-5.10)$ & $\mathrm{NE}$ \\
\hline \multicolumn{6}{|l|}{ Psychosocial/lifestyle factors } \\
\hline \multicolumn{6}{|l|}{ Homosexual experience } \\
\hline Never since age 18 & 10.1 & NA & 1.00 & & Non-significant ${ }^{\mathrm{f}}$ \\
\hline Ever since age 18 & 4.4 & NA & $0.41(0.22-0.78) \dagger$ & & \\
\hline Current job/work satisfaction & & Ns & & & \\
\hline Satisfied & 18.1 & 10.6 & 1.00 & & Non-significant ${ }^{\mathrm{g}}$ \\
\hline Neutral/not sure & 14.9 & 13.5 & $0.79(0.35-1.77)$ & & \\
\hline Dissatisfied & 18.5 & 8.8 & $1.03(0.87-1.21)$ & & \\
\hline \multicolumn{6}{|l|}{ Current financial satisfaction } \\
\hline Satisfied & 29.2 & 21.2 & 1.00 & & 1.00 \\
\hline More or less satisfied & 25.7 & 17.4 & $0.83(0.75-0.94) \dagger$ & & $1.46(1.03-2.06)^{*}$ \\
\hline Not at all satisfied & 24.5 & 14.0 & $0.79(0.70-0.89)^{*}$ & & $1.43(0.97-2.11)^{\S}$ \\
\hline \multicolumn{6}{|l|}{ Social engagement levels } \\
\hline At least several times/month & 25.9 & 16.2 & 1.00 & & Non-significant ${ }^{\mathrm{g}}$ \\
\hline Once/month-several times/yr & 22.1 & 14.5 & $0.81(0.72-0.93) \dagger$ & & \\
\hline Socialize once/year or less & 43.0 & 33.2 & $2.16(1.81-2.57)^{*}$ & & \\
\hline \multicolumn{6}{|l|}{ Sexual attitudes } \\
\hline Liberal attitudes & 13.9 & 5.3 & 1.00 & & 1.00 \\
\hline Moderate attitudes & 15.2 & 7.2 & $1.11(0.64-1.94)$ & & $0.97(0.68-1.26)$ \\
\hline Conservative attitudes & 34.3 & 24.8 & $3.23(1.89-5.52)^{*}$ & & $3.60(1.86-6.96)^{+}$ \\
\hline
\end{tabular}

All statistically significant associations are shown in bold

* $p<.05 ; ;^{\dagger} p<.01 ;{ }^{*} p<.001 ;^{\S} p<.10$

** Adjusted for Year 2000 equivalent value. NE non-estimatable due to small sample size

The following variables were non-significant and dropped from the stepwise regression model for females

${ }^{\mathrm{a}}$ Education: $13-16$ years $=0.95(0.72-1.24)$; high school diploma $=0.87(0.65-1.16) ; 11$ years or less $=0.83(0.60-1.16)$

Contribution to household income: $60-79 \%=0.97(0.62-1.53) ; 40-59 \%=1.07(0.68-1.68) ; 20-39 \%=0.82(0.49-1.37) ; 0-19 \%=0.78(0.52-1.16)$

${ }^{\mathrm{c}}$ Education: 13-16 years $=0.93(0.41-2.12)$; high school diploma $=0.72(0.28-1.81)$; 11 years or less $=0.84(0.26-2.73)$

${ }^{\mathrm{d}}$ Household income: $\$ 60 \mathrm{~K}-74,999=2.10(0.62-7.20) ; \$ 45 \mathrm{~K}-59,999=0.99(0.34-2.95) ; \$ 30 \mathrm{~K}-44,999=2.01(0.75-5.38) ; \$ 15 \mathrm{~K}-29,999=1.33$ $(0.50-3.52) ;<15 \mathrm{~K}=1.32(0.48-3.68)$

e Contribution to household income: $60-79 \%=0.84(0.33-2.15) ; 40-59 \%=1.75(0.75-4.08) ; 20-39 \%=0.90(0.33-2.46) ; 0-19 \%=0.69$ $(0.20-2.39)$

f Job satisfaction: neutral $=0.00(0$-inf $)$; dissatisfied $=1.32(0.71-2.44)$

g Social engagement levels: socialized $1 \times$ month-several times/year $=1.02(0.65-1.59)$; socialized $1 \times /$ year or less $=0.64(0.29-1.42)$

potential confounding effects of age, socioeconomic status, race, and social engagement levels. In particular, never-married adults showed virtually identical levels of happiness between sexually active and sexless participants.

\section{Discussion}

We found that nearly one in six men and over one in four women in the USA did not have sex for at least one year; moreover, the majority of the sexually inactive were sexless for five years or more. Sexual inactivity is thereby not necessarily a short-term interruption of sex, but often represents a long-lasting lifestyle in a non-negligible percentage of American adults. The proportion of individuals who reported past-year sexlessness was roughly similar to those estimated by other large-scale, cross-sectional US studies (Laumann et al., 2009; Smith, 1991) and corroborated the generally higher prevalence among females (Waite, Laumann, Das, \& Schumm, 2009). Due to the lack of reliable population-based data from the international literature, however, it could not be ascertained whether American adults as a whole are more or less sexless than their counterparts in other countries. However, remarkably similar percentage of past-year sexlessness was noted between a Swedish study of 70-year-old adults (34\% of males and $66 \%$ of females) 
Table 5 Self-reported past-year happiness levels versus past-year sexlessness status with multinomial odds ratios of happiness levels among healthy adults $^{\mathrm{a}}$

\begin{tabular}{|c|c|c|c|c|c|c|}
\hline \multirow[t]{2}{*}{ Happiness levels outcomes } & \multicolumn{3}{|l|}{ Males } & \multicolumn{3}{|l|}{ Females } \\
\hline & Had sex & Sexless & $\mathrm{OR}_{\text {adj }}(95 \% \mathrm{CI})^{\mathrm{b}}$ & Had sex & Sexless & $\mathrm{OR}_{\mathrm{adj}}(95 \% \mathrm{CI})^{\mathrm{b}}$ \\
\hline Married participants & $n=2739$ & $n=190$ & & $n=2959$ & $n=183$ & \\
\hline Very happy & $40.5 \%$ & $42.6 \%$ & Referent outcome & $43.0 \%$ & $30.6 \%$ & Referent outcome \\
\hline Pretty happy & $54.1 \%$ & $51.1 \%$ & $1.02(0.61,1.71)$ & $51.5 \%$ & $59.0 \%$ & $0.68(0.40,1.16)$ \\
\hline Not too happy & $5.4 \%$ & $6.3 \%$ & $1.13(0.41,3.14)$ & $5.5 \%$ & $10.4 \%$ & $0.67(0.23,2.00)$ \\
\hline Never-married participants & $n=1094$ & $n=317$ & & $n=1007$ & $n=324$ & \\
\hline Very happy & $20.8 \%$ & $18.9 \%$ & Referent outcome & $23.2 \%$ & $23.8 \%$ & Referent outcome \\
\hline Pretty happy & $65.5 \%$ & $65.9 \%$ & $1.08(0.66,1.78)$ & $62.7 \%$ & $63.3 \%$ & $0.73(0.44,1.20)$ \\
\hline Not too happy & $13.6 \%$ & $15.1 \%$ & $0.93(0.45,1.89)$ & $14.1 \%$ & $13.0 \%$ & $0.61(0.28,1.30)$ \\
\hline Divorced/separated participants & $n=681$ & $n=145$ & & $n=895$ & $n=435$ & \\
\hline Very happy & $17.0 \%$ & $20.7 \%$ & Referent outcome & $18.7 \%$ & $20.9 \%$ & Referent outcome \\
\hline Pretty happy & $66.8 \%$ & $61.4 \%$ & $1.08(0.53,2.19)$ & $64.4 \%$ & $62.1 \%$ & $1.56(0.97,2.50)$ \\
\hline Not too happy & $16.2 \%$ & $17.9 \%$ & $0.65(0.26,1.64)$ & $17.0 \%$ & $17.0 \%$ & $1.36(0.69,2.68)$ \\
\hline Widowed participants & $n=79$ & $n=117$ & & $n=142$ & $n=771$ & \\
\hline Very happy & $30.4 \%$ & $19.7 \%$ & Referent outcome & $26.8 \%$ & $25.3 \%$ & Referent outcome \\
\hline Pretty happy & $58.2 \%$ & $56.4 \%$ & $0.39(0.09,1.64)$ & $48.6 \%$ & $60.6 \%$ & $0.66(0.28,1.53)$ \\
\hline Not too happy & $11.4 \%$ & $23.9 \%$ & $0.52(0.06,4.79)$ & $24.6 \%$ & $14.1 \%$ & $1.54(0.53,4.45)$ \\
\hline
\end{tabular}

a Participants with baseline health reported as "poor" were excluded from analysis

b Adjusted for age (18-34, 35-49, 50-64, 65+), household income, education, race, and social engagement levels (see Table 3 for categories)

(Beckman, Waern, Gustafson, \& Skoog, 2008) with our own study population (41.6\% and 64.8\%, respectively). Middle-aged, married Americans, however, were notably much less likely to be sexless than their counterparts in East Asia. A study of married Hong Kong Chinese between 45 to 59 years of age revealed that onethird of women and $17 \%$ of the males were sexually abstinent (Kim, Lau, \& Cheuk, 2009) (versus $5.7 \%$ and $4.5 \%$, respectively, in our sample). A Japanese study of married couples up (20-59 years of age) also reported that $24 \%$ of the sample did not have sex in the past year (Moriki et al., 2015). The similar percentages of marital sexlessness between genders in our study suggest that extramarital sexual relationships are not strongly skewed toward one sex. The much lower prevalence of marital sexlessness in Americans is likely to be partially attributable to the relatively higher prevalence of divorce in Western countries (18.2\% of this study sample versus $<5 \%$ in the Chinese population) (Hong Kong Government, 2007), suggesting that couples in poor marital relationships in Western countries are less likely to remain together than in countries with lower cultural acceptance of divorce.

Examination of past 5-year sexlessness prevalence among never-married Americans in our study showed high levels of sexual inactivity across all age groups, lending credence to speculations that asexual individuals comprise a non-trivial percentage of the population. Recent research on asexuality has estimated that $1.5 \%$ of males and $3.3 \%$ of females in Finland reported no sexual attraction to others in the past year (Hoglund et al., 2014) and that about $1 \%$ of British adults have never experienced sexual attrac- tion to anyone at all (Bogaert, 2004). The proportion of past 5-year sexlessness among those who have never been married in the 2545 age group in our study was much higher $(8.7 \%$ for males, $11.3 \%$ for females) that the above-cited prevalence estimates for asexuality but not vastly different from a 2002 study of lifetime virgins of the same age group (Eisenberg et al., 2009), suggesting that a large proportion of these individuals with long-term sexual inactivity are likely to have had lifetime abstention from sex. The much higher prevalence of past 5-year sexlessness reported in our study as compared with asexuality estimates, however, suggests that the absence of sexual activity has strong sociological dimensions beyond lack of sexual attraction toward others. The implications of our findings on the phenomenon of asexuality should be interpreted with caution since the absence of sexual activity is not equivalent to asexuality.

Although older age and not being married were by far the strongest factors associated with past-year sexlessness, there were statistically significant psychosocial, economic, and attitudinal factors associated with sexlessness for both genders. For instance, contributing less than $20 \%$ of the household income was a significant correlate of short-term sexlessness for males only, while lower satisfaction with one's financial situation was associated with shortterm sexlessness among females only. This may be indicative of gender power dynamics or psychosocial stressors that may strongly influence sexual opportunities or libido. Similar to studies that found strong associations between lifetime abstinence and religiosity (Eisenberg et al., 2009; Haydon et al., 2014; Matthias et al., 1997), conservative sexual attitudes were associated with past-year sex- 
lessness among females and marginally significant $(p<.10)$ associations among males. Although we did not report the associations of past 5-year sexlessness with the various background factors, due to the temporal instability of many of these variables (e.g., income, self-reported health, and even marital status), the factors associated with past 5-year sexlessness were largely similar to those that were associated with past-year sexlessness. In both sexes, long-term sexlessness was thereby most strongly associated with older age and marital status, whereas socioeconomic factors (e.g., income-related variables), cultural attitudes (conservatism), and fulfillment in other life domains (financial satisfaction) showed less robust associations.

Perhaps most surprising was that sexually inactive people were no less happy than their sexually active counterparts. Most noteworthy, never-married participants showed virtually identical levels of happiness levels regardless of their sexual activity status. Although sexual inactivity among physically healthy adults has often been seen as an indicator of poor emotional well-being in popular psychology (Schnarch \& Maddock, 2003; Shreiner-Engel \& Schiavi, 1986; Weiner-Davis, 2003), a study conducted on a nationally representative sample of American women revealed that the majority of women with low sexual desire (72.5\%) did not report distress over their lack of interest in sex (Rosen et al., 2009). Our results also strongly suggest that sexual activity per se is not a requisite component of emotional well-being. Previous research in the area of psychological well-being and subjective well-being supports the correlation of positive, close relations with others (relatedness) as one of the most important components of human well-being (Argyle, 1987; Myers, 1992). Sexual activity does not capture all romantic feelings, nor does it capture the quality of intimate relationships with others. Based on our study results, there may be other dimensions of close human relationships that are much more integral aspects of well-being and that sexual activity may either be replaced by these other dimensions, or is peripheral to the core areas of emotional wellbeing. The other domains that are common to well-being theories include having control over the course of one's life (autonomy), feeling in control of one's situation (competency/mastery) (Ryan \& Deci, 2001) as well such domains as self-acceptance, life purpose, and personal growth (Ryff \& Keyes, 1995; Ryff \& Singer, 1998), none of which explicitly include sexual activity.

A potential limitation of this study was that it was based upon self-report data and large differences in reporting biases between groups may compromise the validity of the findings. However, validation studies of sexual behaviors have noted high level of accuracy for recalling past-year abstinence (Jaccard et al., 2004). Another limitation of this study is that the GSS-National Death Index 2008 dataset only includes sexual activity data until 2002 and sexual behaviors in the population may have changed since then. However, when we examined the prevalence of sexlessness between 1988 and 2002, there were no significant differences in the prevalence of sexlessness in the population after adjusting for marital status, so any changes since then are likely to be moderate. The GSS-NDI dataset nonetheless contains a large body of information including extensive information about the participants' ideas, beliefs, feelings, and general attitudes, so a broad array of potential confounders were available. Nevertheless, it is possible that unknown confounding variables could have rendered a positive association non-significant. Moreover, the survey determined the sexless status of participants by inquiring about the number of sexual partners and did not ask details about the nature of those sexual partnerships and whether those interactions were voluntary or not. Without detailed information about sexual behaviors, the measure of sexual activity remains quite non-specific. Asking about the number of sexual parthers is also open to interpretation and may not include coitus. A study conducted in 1999 in a U.S. student-aged population revealed that there exist multiple interpretations of having "had sex." While nearly all respondents considered coitus as sex and would include these encounters as sexual partnerships, approximately $40 \%$ considered oral sex alone and $2 \%$ considered "deep kissing" alone as having "had sex" (Sanders \& Reinisch 1999). Nonetheless, using the absence of sexual partners as a definition of sexual inactivity allows for easier cross-cultural comparisons rather than asking about specific sexual behaviors such as oral sex which may not be culturally acceptable for population-based surveys in many parts of the world. Lastly, a limitation of this study was the scale for measuring happiness. Although this construct was measured with a single question and broadly classified people into three happiness levels, we noted that this variable correlated significantly in a small subset of individuals $(n=1170)$ who were asked about their emotional well-being in past month. Happiness showed strong inverse relationships with feelings of cheerlessness, nervousness, hopelessness, and worthlessness $(p<.001)$. When we looked at the correlations between self-reported happiness with job satisfaction, financial satisfaction, and satisfaction with non-work activities, the data also indicated a strong positive association $(p<.001)$, suggesting that a single-item happiness scale adequately captures this very abstract construct.

Our findings contradict the perception promulgated by popular media that the absence of partnered sexual activity commonly results in poorer happiness levels. The lack of difference in happiness levels between sexless and sexually active respondents, particularly among never-married individuals, indicates the need to re-examine the relative importance of sexual activity to overall well-being over an individual's life course. This study's findings also support the DSM-5 revisions for the diagnosis of sexual dysfunctions that now require significant emotional distress as a diagnostic criterion. Given the surprisingly high proportion of Americans that report both short-term and long-term sexlessness, future research should investigate the self-reported reasons for the absence of sexual activity among these adults.

Acknowledgements This study was funded by the National Institutes of Health (5 R21 HD075664-02).

\section{Compliance with Ethical Standards}

Conflict of interest The authors have no conflicts of interest to declare. 
Ethical Approval The General Social Survey-National Death Index was approved by the Columbia University Medical Center Institutional Review Board.

\section{References}

Argyle, M. (1987). The psychology of happiness. London: Methuen.

Beckman, N., Waern, M., Gustafson, D., \& Skoog, I. (2008). Secular trends in self reported sexual activity and satisfaction in Swedish 70 year olds: Cross sectional survey of four populations, 1971-2001. British Medical Journal, 337, a279.

Blanchflower, D. G., \& Oswald, A. J. (2004). Money, sex and happiness: An empirical study. Scandinavian Journal of Economics, 106, 393-415.

Bogaert, A. F. (2004). Asexuality: Prevalence and associated factors in a national probability sample. Journal of Sex Research, 41, 279-287.

Bogaert, A.F.(2015). Asexuality: What it is and why it matters. Journal of Sex Research, 52, 362-379.

Centers for Disease Control and Prevention. (2002). Trends in sexual risk behaviors among high school students-United States, 1991-2001. Journal of the American Medical Association, 288, 1842-1844.

Chou, K. L., Ng, I. S. F., \& Yu, K. M. (2014). Lifetime abstention of sexual intercourse and health in middle-aged and older adults: Results from Wave 2 of the National Epidemiologic Survey on Alcohol and Related Conditions. Archives of Sexual Behavior, 43, 891-900.

DeLamater, J., \& Moorman, S. M. (2007). Sexual behavior in later life. Journal of Aging and Health, 19, 921-945.

DeVellis, R. (1991). Scale development. Newbury Park, CA: Sage.

Dunn, K. M., Croft, P. R., \& Hackett, G. I. (1999). Association of sexual problems with social, psychological, and physical problems in men and women: A cross sectional population survey. Journal of Epidemiology and Community Health, 53, 144-148.

Eisenberg, M. L., Shindel, A. W., Smith, J. F., Breyer, B. N., \& Lipshultz, L. I. (2010). Socioeconomic, anthropomorphic, and demographic predictors of adult sexual activity in the United States: Data from the National Survey of Family Growth. Journal of Sexual Medicine, 7, 50-58.

Eisenberg, M. L., Shindel, A. W., Smith, J. F., Lue, T. F., \& Walsh, T. J. (2009). Who is the 40-year-old virgin and where did he/she come from? Data from the National Survey of Family Growth. Journal of Sexual Medicine, 6, 2154-2161.

Finer, L. B. (2007). Trends in premarital sex in the United States, 1954-2003. Public Health Reports, 122, 73-78.

Haydon, A. A., Cheng, M. M., Herring, A. H., McRae, A. L., \& Halpern, C. T. (2014). Prevalence and predictors of sexual inexperience in adulthood. Archives of Sexual Behavior, 43, 221-230.

Heiman, J. R., Long, J. S., Smith, S. N., Fisher, W. A., Sand, M. S., \& Rosen, R. C. (2011). Sexual satisfaction and relationship happiness in midlife and older couples in five countries. Archives of Sexual Behavior, 40, 741-753.

Herbenick, D., Reece, M., Schick, V., Sanders, S. A., Dodge, B., \& Fortenberry, J. D. (2010). Sexual behaviors, relationships, and perceived health status among adult women in the United States: Results from a national probability sample. Journal of Sexual Medicine, 7(Suppl. 5), 277-290.

Hoglund, J., Jern, P., Sandnabba, N. K., \& Santtila, P. (2014). Finnish women and men who self-report no sexual attraction in the past 12 months: Prevalence, relationship status and sexual behavior history. Archives of Sexual Behavior, 43, 879-889.

Hong Kong Government. (2007). Marriage and divorce trends in Hong Kong, 1981 to 2006. Accessed November 15, 2015. Available from: http://www.statistics.gov.hk/pub/B70711FB2007XXXXB0100.pdf.

Jaccard, J., McDonald, R., Wan, C. K., Guilamo-Ramos, V., Dittus, P., \& Quinlan, S. (2004). Recalling sexual partners: The accuracy of selfreports. Journal of Health Psychology, 9, 699-712.

Kim, J. H., Lau, J. T., \& Cheuk, K. K. (2009). Sexlessness among married Chinese adults in Hong Kong: Prevalence and associated factors. Journal of Sexual Medicine, 6, 2997-3007.
Laumann, E. O., Glasser, D. B., Neves, R. C., \& Moreira, E. D. (2009). A population-based survey of sexual activity, sexual problems and associated help-seeking behavior patterns in mature adults in the United States of America. International Journal of Impotence Research, 21, 171-178.

Laumann, E. O., Nicolosi, A., Glasser, D. B., Paik, A., Gingell, C., Moreira, E., et al. (2005). Sexual problems among women and men aged 40-80y: Prevalence and correlates identified in the Global Study of Sexual Attitudes and Behaviors. International Journal of Impotence Research, 17, 39-57.

Lindau, S. T., \& Gavrilova, N. (2010). Sex, health, and years of sexually active life gained due to good health: Evidence from two US population based cross sectional surveys of ageing. British Medical Journal, 340, c810.

Lindau, S. T., Schumm, L. P., Laumann, E. O., Levinson, W., O'Muircheartaigh, C. A., \& Waite, L. J. (2007). A study of sexuality and health among older adults in the United States. New England Journal of Medicine, 357, 762-774.

Marsiglio, W., \& Donnelly, D. (1991). Sexual relations in later life: A national study of married persons. Journal of Gerontology, 46, S338-344.

Matthias, R. E., Lubben, J. E., Atchison, K. A., \& Schweitzer, S. O. (1997). Sexual activity and satisfaction among very old adults: Results from a community-dwelling Medicare population survey. The Gerontologist, 37, 6-14.

Moriki, Y., Hayashi, K., \& Matsukura, R. (2015). Sexless marriages in Japan: Prevalence and reasons. In N. Ogawa \& I. H. Shah (Eds.), Low fertility and reproductive health in East Asia (pp. 161-185). New York: Springer.

Muennig, P., Johnson, G., Kim, J., Smith, T. W., \& Rosen, Z. (2011). The General Social Survey-National Death Index: An innovative new dataset for the social sciences. BMC Research Notes, 4, 385.

Myers, D. G. (1992). The pursuit of happiness. Who is happy and why. New York: William Morrow.

Nettleman, M., Ingersoll, K. S., \& Ceperich, S. D. (2006). Characteristics of adult women who abstain from sexual intercourse. Journal of Family Planning and Reproductive Health Care, 32, 23-24.

Nicolosi, A., Buvat, J., Glasser, D. B., Hartmann, U.,Laumann, E. O., Gingell, C., et al. (2006). Sexual behaviour, sexual dysfunctions and related help seeking patterns in middle-aged and elderly Europeans: The Global Study of Sexual Attitudes and Behaviors. World Journal of Urology, 24, 423-428.

Nicolosi, A., Glasser, D. B., Kim, S. C., Marumo, K., \& Laumann, E. O. (2005). Sexual behaviour and dysfunction and help-seeking patterns in adults aged 40-80 years in the urban population of Asian countries. BJU International, 95, 609-614.

Palacios-Cena, D., Carrasco-Garrido, P., Hernandez-Barrera, V., AlonsoBlanco, C., Jimenez-Garcia, R., \& Fernandez-de-las-Penas, C. (2012). Sexual behaviors among older adults in Spain: Results from a population-based national sexual health survey. Journal of Sexual Medicine, 9, 121-129.

Pangman, V. C., \& Seguire, M. (2000). Sexuality and the chronically ill older adult: A social justice issue. Sexuality and Disability, 18, 49-59.

Patel, D., Gillespie, B., \& Foxman, B. (2003). Sexual behavior of older women: Results of a random-digit-dialing survey of 2000 women in the United States. Sexually Transmitted Diseases, 30, 216-220.

Prigerson, H. G., Maciejewski, P. K., \& Rosenheck, R. A. (1999). The effects of marital dissolution and marital quality on health and health service use among women. Medical Care, 37, 858-873.

Rasberry, C. N., \& Goodson, P. (2009). Predictors of secondary abstinence in U.S. college undergraduates. Archives of Sexual Behavior, 38, 74 86.

Rosen, R. C., Shifren, J. L., Monz, B. U., Odom, D. M., Russo, P. A., \& Johannes, C. B. (2009). Correlates of sexually related personal distress in women with low sexual desire. Journal of Sexual Medicine, 6, 15491560. 
Ryan, R. M., \& Deci,E. L. (2001). Onhappiness and human potential: A review of research on hedonic and eudaimonic well-being. Annual Review of Psychology, 52, 141-166.

Ryff, C. D., \& Keyes, C. L. M. (1995). The structure of psychological wellbeing revisited. Journal of Personality and Social Psychology, 69, 719-727.

Ryff, C. D., \& Singer, B. (1998). The contours of positive human health. Psychological Inquiry, 9, 1-28.

Sanders, S. A., \& Reinisch, J. M. (1999). Would you say you "had sex" if...? Journal of the American Medical Association, 281, 275-277.

Sandfort, T. G., Orr, M., Hirsch, J. S., \& Santelli, J. (2008). Long-term health correlates of timing of sexual debut: Results from a national US study. American Journal of Public Health, 98, 155-161.

Schick, V., Herbenick, D., Reece, M., Sanders, S. A., Dodge, B., Middlestadt, S. E., et al. (2010). Sexual behaviors, condom use, and sexual health of Americans over 50: Implications for sexual health promotion for older adults. Journal of Sexual Medicine, 7(Suppl. 5), 315-329.

Schnarch, D., \& Maddock, J. (2003). Resurrecting sex: Solving sexual problems and revoloutionizing your relationship. New York: Harper Collins Inc.

Schreiner-Engel, P., \& Schiavi, R. C. (1986). Lifetime psychopathology in individuals with low sexual desire. The Journal of Nervous and Mental Disease, 174, 646-651.

Seligman, M. E. P., \& Csikszentmihalyi, M. (2000). Positive psychology: An introduction. American Psychologist, 55, 5-14.

Seligman, M. E. P., Steen, T. A., Park, N., \& Peterson, C. (2005). Positive psychology progress: Empirical validation of interventions. American Psychologist, 60, 410-421.
Shifren, J. L., Monz, B. U., Russo, P. A., Segreti, A., \& Johannes, C. B. (2008). Sexual problems and distress in United States women: Prevalence and correlates. Obstetrics and Gynecology, 112, 970-978.

Smith, T. W. (1991). Adult sexual behavior in 1989: Number of partners, frequency of intercourse and risk of AIDS. Family Planning Perspectives, 23, 102-107.

Smith, T. W. (2006). American sexual behavior: Trends, socio-demographic differences, and risk behavior (GSS Topical Report No. 25). National Opinion Research Center, University of Chicago. Retrieved from http://www.norc.org/PDFs/Publications/American SexualBehavior2006.pdf.

Turner, C. F., Danella, R. D., \& Rogers, S. M. (1995). Sexual behavior in the United States 1930-1990: Trends and methodological problems. Sexually Transmitted Diseases, 22, 173-190.

Waite, L. J., Laumann, E. O., Das, A., \& Schumm, L. P. (2009). Sexuality: Measures of partnerships, practices, attitudes, and problems in the National Social Life, Health, and Aging Study. Journals of Gerontology, Series B, Psychological Sciences and Social Sciences, 64(Suppl. 1), $56-66$.

Weiner-Davis, M. (2003). The sex-starved marriage. New York: Simon $\&$ Schuster.

Wong, S. Y., Leung, J. C., \& Woo, J. (2009). Sexual activity, erectile dysfunction and their correlates among 1566 older Chinese men in Southern China. Journal of Sexual Medicine, 6, 74-80. 